\title{
Micromachined Gyroscope Design Allowing for Both Robust Wide-Bandwidth and Precision Mode-Matched Operation
}

\author{
Adam R. Schofield, Alexander A. Trusov, and Andrei M. Shkel \\ Dept. of Mechanical and Aerospace Engineering, University of California, Irvine, Irvine, CA, 92697 \\ Email: adam.schofield@uci.edu, atrusov@uci.edu, ashkel@uci.edu
}

\begin{abstract}
This paper presents a new z-axis gyroscope design with a 2-degree of freedom (DOF) sense mode allowing interchangeable operation in either precision (mode-matched) or robust (wide-bandwidth) modes. This is accomplished using a complete 2-DOF coupled system which, unlike the previous multi-DOF design, allows for the specification of the sense mode resonant frequencies and coupling independent of frequency. The robust mode corresponds to operation between the 2-DOF sense mode resonant frequencies providing a response gain and bandwidth controlled by frequency spacing. Precision mode of operation, however, relies on mode-matching the drive to the second, anti-phase sense mode resonant frequency which can be designed to provide a gain advantage over a similar 1-DOF system. Experimental rate characterization of an SOI prototype in air for both robust and precision modes revealed scale factors of 0.282 and $0.690 \mathrm{mV} / \mathrm{deg} / \mathrm{s}$ respectively. The improvement due to precision mode operation is increased to 27 times for operation in $500 \mathrm{mTorr}$ reduced pressure.
\end{abstract}

\section{INTRODUCTION}

Micromachined vibratory gyroscopes operate based on the Coriolis effect where a rotation induced energy transfer occurs between two orthogonal vibrational modes, commonly referred to as drive and sense [1]. Conventionally, these modes are realized as single degree of freedom (DOF) dynamic systems with their own associated resonant frequencies giving rise to two differing methods of operation: mode-matched or mismatched [2]. In mode-matched devices, the drive and sense resonant frequencies are equal allowing the output of the sensor to be increased proportional to the sense mode quality factor yielding higher sensitivities at the cost of reduced bandwidth and robustness [3]. Operation with the resonant frequencies separated by some prescribed amount, or mode-mismatched, is more common, particularly for automotive applications where robustness is critical [4], [5].

Previously, a gyroscope design concept was introduced aimed at robust operation using an expanded sense mode design space through increased degrees of freedom [6]. Specifically, the device used two coupled sense masses forming a 2-DOF dynamic system with two sense mode resonant frequencies and a wide region of constant amplitude between them. While the gain and the bandwidth of this operational region is controlled solely by the resonant frequency spacing, a constraint limited the minimal achievable spacings as the operational frequency of device with fixed size was increased

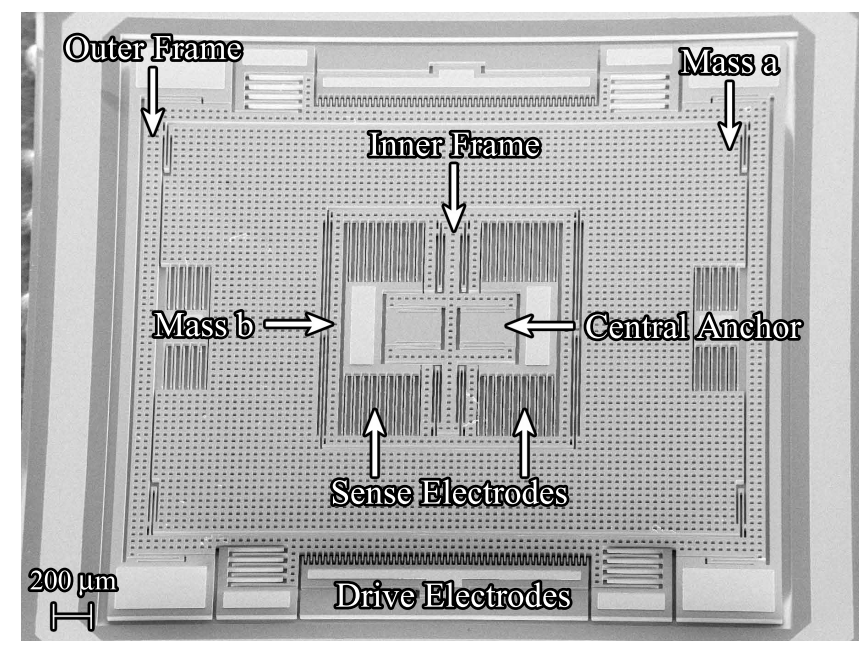

Fig. 1. SEM image of fabricated gyroscope prototype.

[7]. This is a direct effect of the 2-DOF design which utilized only two suspensions thereby eliminating the ability to independently define the frequency spacing and the coupling between the masses. In contrast, a complete 2-DOF system consisting of two masses and three suspensions alleviates this issue allowing for the arbitrary specification of frequency spacing independent of operational frequency [8].

This work presents a new, z-axis micromachined gyroscope design, Fig. 1, for the first time. It takes advantage of a complete 2-DOF coupled sense mode allowing for independent adjustment of both the sense mode resonant frequencies and the amount of coupling. Furthermore, the complete 2-DOF sense mode enables a new operational method where the drive mode can be interchangeably placed between the sense mode peaks (robust mode) or mode-matched to the anti-phase resonant frequency of the 2-DOF sense mode (precision mode). The design concept, described in Section II and characterized in Section III, allows independent control over the gain of both operational modes so the sensor can be tailored for the specific application.

\section{DESIGN CONCEPT}

A lumped structural schematic of the new gyroscope concept is presented in Fig. 2. The design, like previous multi- 


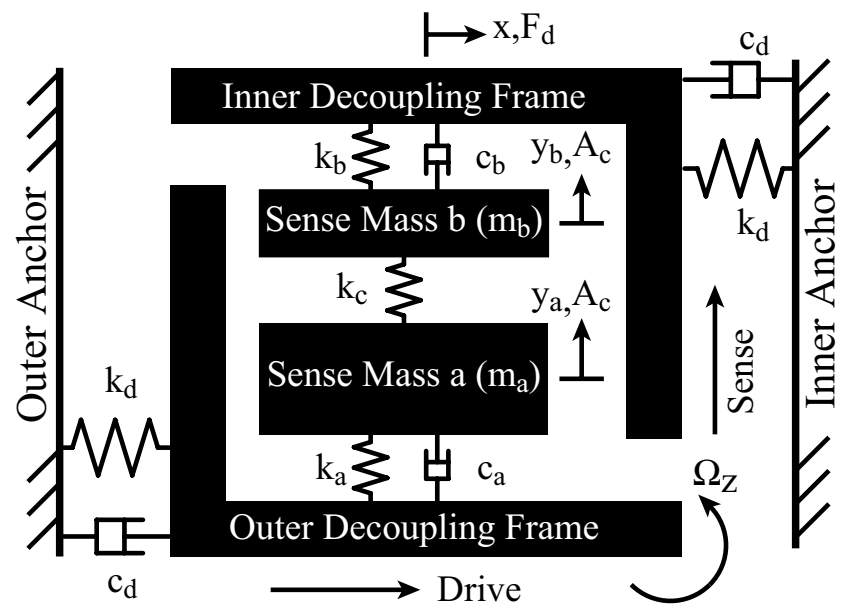

Fig. 2. Lumped structural model of the gyroscope concept.

DOF sense mode devices, consists of a conventional drive mode that is mechanically decoupled from a 2-DOF sense mode dynamic system formed by the two coupled sense masses, $m_{a}$ and $m_{b}$. The major difference compared to the previous multi-DOF implementation [6], however, is the inclusion of a third suspension element in the sense mode forming a complete 2-DOF coupled system. Due to the frame decoupling and symmetry of the design, the addition of this suspension element is possible through the use of a second, inner decoupling frame suspended in the drive mode relative to a central anchor.

Since the drive mode of the presented concept is a conventional 1-DOF resonant system, the desired operational frequency, $\omega_{d}$, can be obtained independent of the sense mode through adjustment of the drive suspension or mass of the decoupling frames. On the other hand, the sense mode is a coupled 2-DOF system where the natural frequencies are determined by eigenvalues in terms of the structural frequencies $\omega_{a}^{2}=\left(k_{a}+k_{c}\right) / m_{a}$ and $\omega_{b}^{2}=\left(k_{b}+k_{c}\right) / m_{b}$, as well as the coupling between the masses, $\omega_{c}^{2}=k_{c} / \sqrt{m_{a} m_{b}}$. If the desired sense mode resonant frequencies, $\omega_{1,2}$ are specified in terms of the drive frequency, $\omega_{d}$, and the sense mode frequency spacing, $\Delta=\omega_{2}-\omega_{1}$, the following sense mode design equations can be found,

$$
\omega_{a, b}^{2}=\omega_{d}^{2}+\left(\frac{\Delta}{2}\right)^{2} \pm \sqrt{\omega_{d}^{2} \Delta^{2}-\omega_{c}^{4}}
$$

which assumes the drive is equally spaced from the sense mode resonances.

As shown in (1), the amount of coupling between the masses, $\omega_{c}^{2}$, can be adjusted independently from the structural frequencies. There is, however, a limited range of values that can be specified while maintaining a physically meaningful system. Therefore, the coupling can be expressed in terms of a percentage of the maximum coupling,

$$
\omega_{c}^{2}=\epsilon \omega_{d} \Delta, \text { where } 0<\epsilon<1,
$$

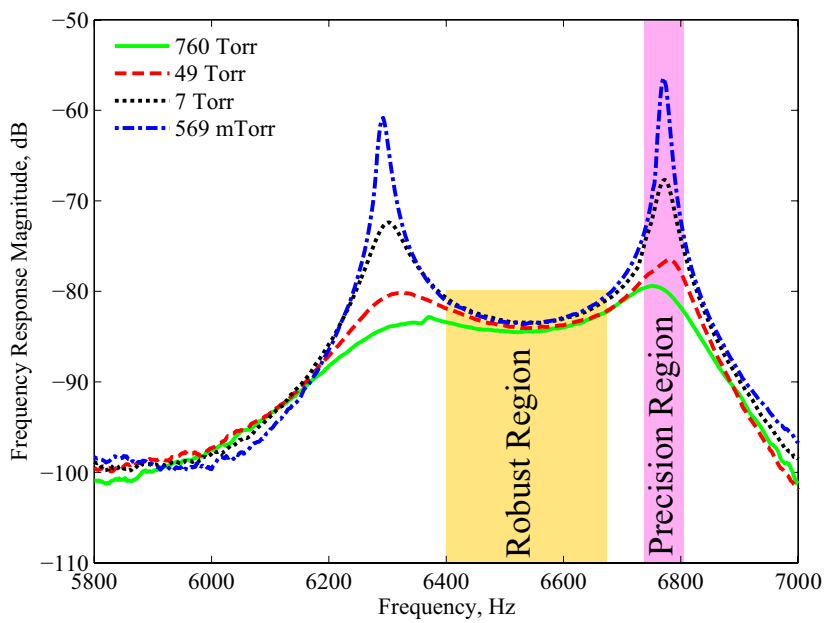

Fig. 3. Experimental sense mode frequency response of a prototype with both the robust and precision operational regions highlighted.

where $\epsilon$ is the coupling parameter. Substituting (2) into (1) gives,

$$
\omega_{a, b}^{2}=\omega_{d}^{2}+\left(\frac{\Delta}{2}\right)^{2} \pm \omega_{d} \Delta \sqrt{1-\epsilon^{2}},
$$

which are the sense mode design equations in terms of desired operational frequency, $\omega_{d}$, sense mode resonant frequency spacing, $\Delta$, and the amount of coupling, $\epsilon$. From (3) and (2), the stiffnesses required to achieve the desired parameters can be found assuming the value of the sense masses are known.

The complete 2-DOF sense mode provides the device with distinct advantages over the previous multi-DOF design which relied on only two suspensions [6]. This includes the ability to achieve the desired sense mode resonant frequencies independent of operational frequency as well as control over the degree of coupling between the sense masses. While the design removes a constraint with earlier implementations [7], it also introduces a new operational method for multi-DOF gyroscopes: interchangeable operation in both precision and robust modes in a single device.

The concept of both robust and precision modes of operation is shown in Fig. 3 which presents frequency response curves of a complete 2-DOF sense mode test structure at various pressures. Similar to previous multi-DOF devices, the robust mode corresponds to operation in the region between the sense mode resonant frequencies; precision operation, however, consists of mode-matching the drive to the sense mode anti-phase resonant frequency. As with conventional devices, the resonant amplitude of the precision mode can be increased with decreasing pressures resulting in higher sensitivities whereas the amplitude of the robust region is mainly a function of resonant frequency spacing. Thus, the gains of each mode can be controlled independently enabling both high sensitivity and robust, wide-bandwidth operation at reduced pressures.

Another advantage of the new design is that increased resonant gain over an equivalent $1-\mathrm{DOF}$ sense mode can be 


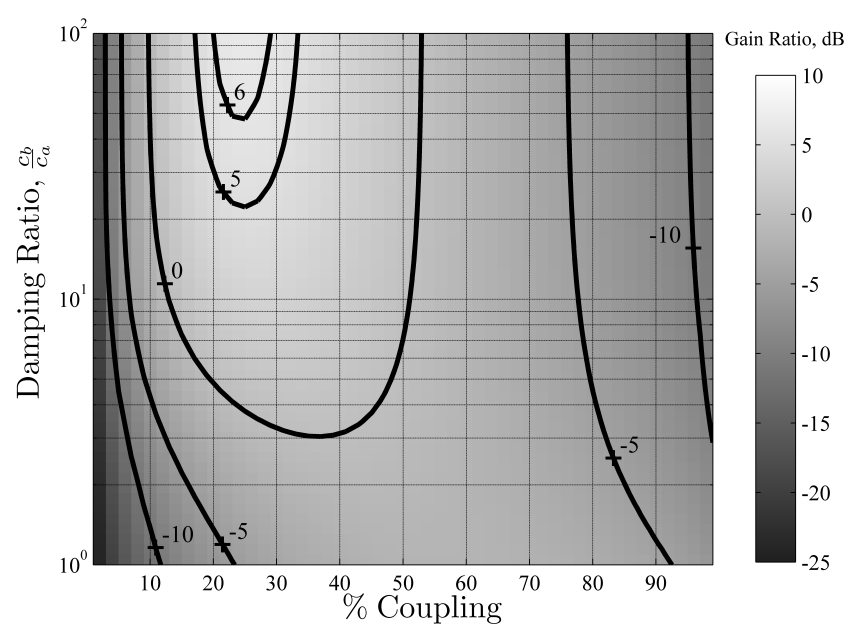

Fig. 4. Gain ratio of 2-DOF versus 1-DOF sense modes as a function of damping ratio and degree of coupling.

obtained for similar damping conditions. This is illustrated with the simulation results in Fig. 4 showing the gain ratio in $\mathrm{dB}$ between the 2-DOF anti-phase resonant frequency and a 1-DOF system of $m_{a}+m_{b}$ at the same frequency. In the simulation, the total damping $c_{a}+c_{b}$, corresponding to a 1-DOF $\mathrm{Q}$ factor of 500, remained fixed for changes in the coupling parameter, $\epsilon$, and the 2-DOF damping ratio, $\frac{c_{b}}{c_{a}}$. There exists design parameters where the 2-DOF system has over a 6 $\mathrm{dB}$ gain advantage versus the equivalent $1-\mathrm{DOF}$ system. This advantage is achieved with larger damping on the detection mass $m_{b}$, meaning that increasing the sensing capacitance, i.e. more sensing plates or smaller capacitive gaps, is advantageous for the presented design.

\section{EXPERIMENTAL RESULTS}

\section{A. Fabrication}

The device used for the experimental characterization presented below was fabricated using an in-house, two mask, wafer scale SOI process with a conductive $50 \mu \mathrm{m}$ device layer and a $5 \mu \mathrm{m}$ buried oxide. First, a front side metalization process defined bonding pads via lift-off followed by a Deep Reactive Ion Etching (DRIE) step using a Surface Technology Systems (STS) Advanced Silicon Etching (ASE) tool. The minimum feature size of the overall process used to define the capacitive gaps was $5 \mu \mathrm{m}$. The perforated structures were released using a timed HF etch followed by dicing, packaging, and wire bonding. An SEM image of a fabricated gyroscope prototype is presented in Fig. 1.

\section{B. Biasing Scheme}

Fig. 5 presents a schematic of the actuation and detection scheme used in the experimental characterization below. For drive mode actuation, an AC driving voltage, $\nu_{d}(t)$, plus a DC potential, $V_{d}$, was applied to the fixed electrodes while a high frequency AC carrier voltage, $\nu_{c}(t)$, plus a sense mode tuning voltage, $V_{t}$, was applied to the mobile mass. In

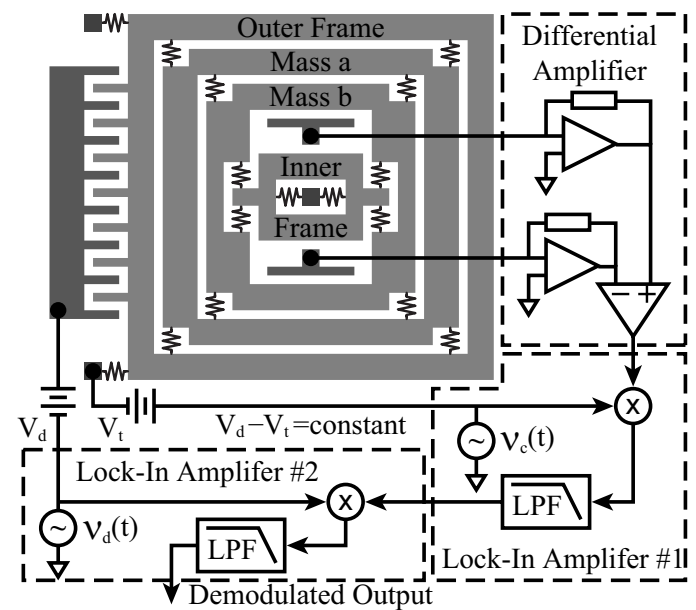

Fig. 5. Schematic of the actuation and detection architecture used for the experimental characterization of the gyroscope.

order to maintain a constant driving amplitude, the total DC driving potential, $V_{d}-V_{t}$, was fixed during sense mode tuning experiments.

In the sense mode, a differential detection scheme using cascaded trans-impedance and instrumentation amplifiers was used to pick up the motion of mass $m_{b}$. The differential signal was then demodulated at the carrier frequency followed by a second demodulation at the drive frequency using AMTEK Model 7265 Lock-In Amplifiers. The use of the high frequency carrier allows for the separation of useful sense signals from parasitics [9].

\section{Mode-Matching}

The presented device was designed with the drive mode natural frequency of $5.44 \mathrm{kHz}$ between the lower and higher sense mode resonances of 5.2 and $5.6 \mathrm{kHz}$ respectively. Therefore, mode-matching for the presented device required electrostatic tuning of the sense mode in order to match the higher anti-phase resonant frequency to the drive. By adjusting the DC tuning voltage, $V_{t}$, applied to the mass, the sense mode resonant frequencies can be shifted down in frequency as shown in the inset of Fig. 6. As the voltage is increased, both sense mode frequencies are shifted until the higher sense mode frequency crosses the drive mode at approximately 35 $\mathrm{V}$.

While the sense mode tuning curves indicate that the device can be mode-matched, it does not ensure precise matching is achieved. The zero rate output of the gyroscope, or quadrature, however, can be monitored for changes in tuning voltage with a maximum output occurring when the drive and sense frequencies are equal [10]. The quadrature output in $\mathrm{dB}$ versus tuning voltage is presented in Fig. 6 for the device operated in 5.6 Torr vacuum. For low tuning voltages $(0-15 \mathrm{~V})$, the drive mode is in the robust operational mode between the peaks; as the voltage is increased, the quadrature signal increases until a maximum is achieved at $34 \mathrm{~V}$ indicating a mode-matched 


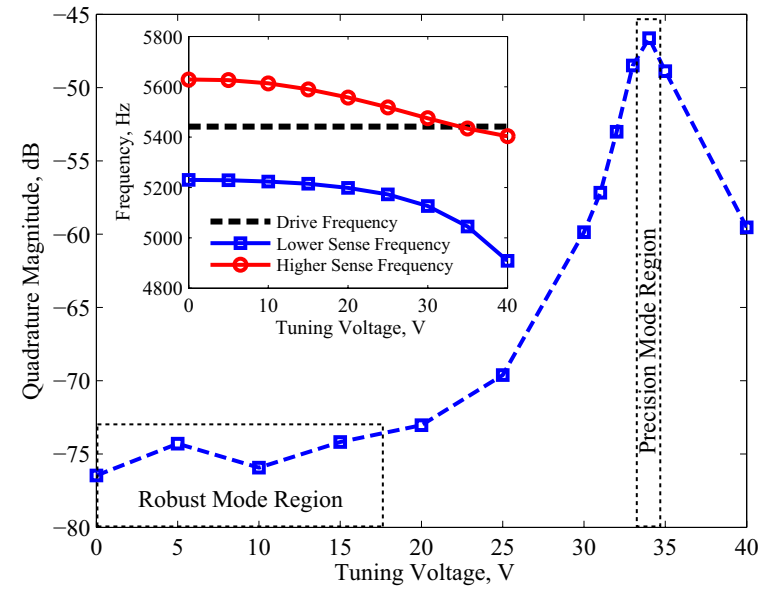

Fig. 6. Zero rate output of the gyroscope as a function of tuning voltage; inset: sense mode resonant frequencies versus applied tuning voltage.

condition, while even higher voltages result in decreasing signals.

\section{Angular Rate Characterization}

To verify both operational modes, the device was characterized in atmospheric pressure at constant angular rates using an Ideal Aerosmith 1291BR rate table. The actuation and detection scheme shown in Fig. 5 was used with tuning voltages of $0 \mathrm{~V}$ and $34 \mathrm{~V}$ for robust and precision modes, respectively, while the total driving voltage was kept constant. For robust operation, the experimentally obtained scale factor was $0.282 \mathrm{mV} / \mathrm{deg} / \mathrm{s}$ while for precision operation it was $0.690 \mathrm{mV} / \mathrm{deg} / \mathrm{s}$. Thus, precision mode resulted in a 2.4 times improvement in scale factor at atmospheric pressure, which is calculated to rise 27 times for operation in 500 mTorr vacuum from Fig. 3 .

\section{CONCLUSIONS}

A new z-axis gyroscope concept with a complete 2-DOF sense mode was presented. The device, unlike the previous multi-DOF design, allows for the specification of the sense mode frequency spacing and coupling independent of the operational frequency through the addition of a second, inner decoupling frame. Additionally, a new operational method was introduced allowing interchangeable operation in either precision (mode-matched) or robust (wide-bandwidth) modes. The robust mode corresponds to operation between the 2-DOF sense mode resonant frequencies providing a robust response gain controlled by the frequency spacing. Precision operation, however, relies on mode-matching the drive to the sense mode anti-phase resonant frequency so that the amplitude increases with decreasing pressures. Also, for specific design parameters, a gain advantage over a similar 1-DOF system can be achieved. Experimental rate characterization of an SOI prototype operated in both robust and sensitive modes revealed a 2.4 times scale factor improvement at atmospheric pressure which can be increased 27 times for 500 mTorr pressure.

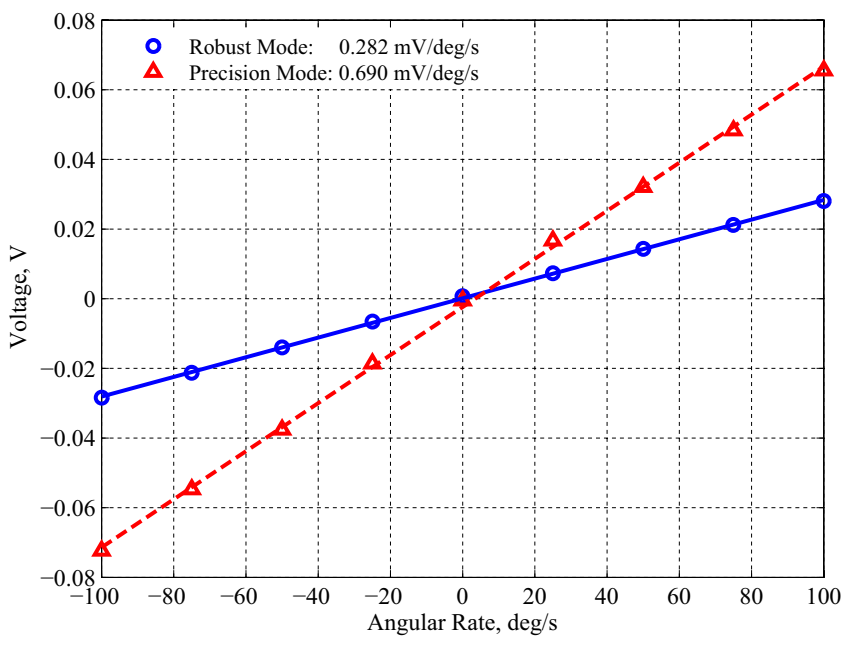

Fig. 7. Constant angular rate characterization for both robust and precision modes in air.

\section{ACKNOWLEDGMENTS}

This work was partially supported by the National Science Foundation Grant CMS-0409923, Custom Sensors \& Technologies Systron Donner Automotive (formerly BEI Technologies) contract BEI-36974, and UC Discovery program ELE0410202. The gyroscopes were fabricated at the UCI Integrated Nanosystems Research Facility (INRF) and experimental characterization was performed at the UCI MicroSystems Laboratory. The authors would like to thank the Carl Zeiss Center of Excellence for use of the Ultra 55 SEM.

\section{REFERENCES}

[1] A. M. Shkel, "Type I and Type II micromachined vibratory gyroscopes," in Proc. of IEEE/ION PLANS, San Diego, CA, USA, Apr. 24-27, 2006.

[2] M. Weinberg and A. Kourepenis, "Error sources in in-plane silicon tuning-fork MEMS gyroscopes," J. Microelectromech. Syst., vol. 15, no. 3, pp. 479-491, June 2006.

[3] N. Yazdi, F. Ayazi, and K. Najafi, "Micromachined inertial sensors," Proc. IEEE, vol. 86, no. 8, pp. 1640-1659, 1998.

[4] R. Neul, U.-M. Gomez, K. Kehr, W. Bauer, J. Classen, C. Doring, E. Esch, S. Gotz, J. Hauer, B. Kuhlmann, C. Lang, M. Veith, and R. Willig, "Micromachined angular rate sensors for automotive applications," IEEE Sensors J., vol. 7, no. 2, pp. 302-309, Feb. 2007.

[5] J. A. Geen, S. J. Sherman, J. F. Chang, and S. R. Lewis, "Singlechip surface micromachined integrated gyroscope with $50 \mathrm{deg} / \mathrm{h}$ Allan deviation," IEEE J. Solid-State Circuits, vol. 37, no. 12, pp. 1860-1866, 2002.

[6] C. Acar and A. Shkel, "Inherently robust micromachined gyroscopes with 2-DOF sense-mode oscillator," J. Microelectromech. Syst., vol. 15, no. 2, pp. 380-387, Apr. 2006.

[7] A. R. Schofield, A. A. Trusov, and A. M. Shkel, "Effects of operational frequency scaling in multi-degree of freedom MEMS gyroscopes," IEEE Sensors J., 2008, accepted for publication.

[8] A. A. Trusov, A. R. Schofield, and A. M. Shkel, "New architectural design of a temperature robust MEMS gyroscope with improved gainbandwidth characteristics," in Proc. of the 2008 Hilton Head SSSAMW, Hilton Head, SC, USA, June 1-5, 2008.

[9] A. A. Trusov and A. M. Shkel, "Capacitive detection in resonant MEMS with arbitrary amplitude of motion," J. Micromech. Microeng., vol. 17, no. 8, pp. 1583-1592, July 2007.

[10] A. Sharma, M. Zaman, and F. Ayazi, "A smart angular rate sensor system,” in Proc. IEEE Sensors, Atlanta, GA, USA, Oct. 28-31, 2007, pp. 1116-1119. 